\title{
Using Back Propagation Neural Network for Cardiac Wall Motion Detection on Angiocardiography
}

\author{
Yu-Chien Shiau ${ }^{1,2}$, Hsiao-Hsuan Chou ${ }^{1}$, Shue-Tsun Fan ${ }^{3}$, \\ Shu-Hsun $\mathrm{Chu}^{4}$, Te-Son Kuo ${ }^{1,5}$ \\ ${ }^{I}$ Department of Electrical Engineering, ${ }^{5}$ Institute of Biomedical Engineering, \\ National Taiwan University; ${ }^{3}$ National Taiwan Normal University; ${ }^{2}$ Department of \\ Nuclear Medicine, ${ }^{4}$ Cardiovascular Center, Far Eastern Memorial Hospital, Taiwan \\ E-mail: helloshiau@mail.femh.org.tw
}

\begin{abstract}
We used back propagation neural network for cardiac wall motion detection on catheterization angiocardiography. Phantom images of two circular discs were generated to simulate the left ventricle. Training data sets were selected from the phantom images. After training, the neural network can perform cardiac wall motion detection for the phantom images and for all series of patients' angiocardiographic images. The angiocardiography of one patient with normal cardiac wall motion and two patients with abnormal cardiac wall motion were acquired for cardiac wall motion detection. The results were displayed in the formats of vector fields superimposed on the original angiocardiographic images. The study showed that back propagation neural network was useful in the evaluation of cardiac wall motion on catheterization angiocardiography.
\end{abstract}

\section{Introduction}

Cardiac wall motion detection is an important analysis of various kinds of cardiac images, such as catheterization contrast angiocardiography, cardiac ultrasonography, and cardiac cine MRI. The analysis is useful for the clinical diagnosis of various heart diseases. The conventional approaches for motion detection were matching, gradient methods, pattern recognition, temporal texture, optical flow methods [1], block-based methods, pel-recursive methods, and Bayesian methods [2]. Motion detection by neural networks is a relatively new method, and has become more successful in various kinds of simulation studies. Local motion detection and its extension for arbitrary motion detection were achieved by back propagation neural network [3]. Detection of moving and standing objects was achieved by cellular neural networks [4]. In our previous study, the neural network was useful in cardiac wall motion detection of Tc-99m MIBI myocardial GSPECT [5]. The catheterization contrast angiocardiography is an important imaging modality for the evaluation of cardiac wall motion and very useful in the clinical study of various heart diseases [6]. In this study we used back propagation neural network for cardiac wall motion detection on angiocardiography.

\section{Material and methods}

\subsection{Back propagation neural network}

We used a three-layer back propagation neural network for motion detection (Fig. 1). For any arbitrary center pixel $(i, j)$ with its surrounding 8 pixels, the 9 pixels form a $3 \times 3$ 


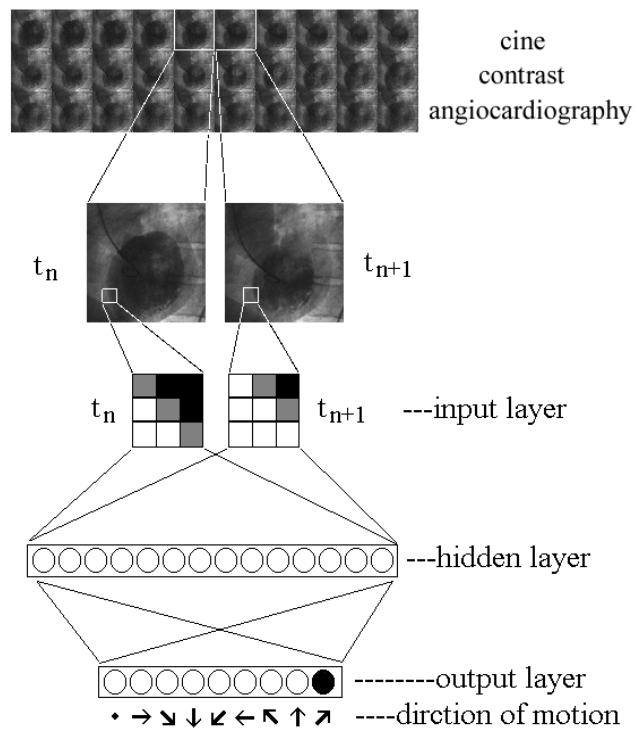

Figure 1. Three-layer back propagation neural network consists of 18 nodes in input layer and 9 nodes in output layer.

matrix. The brightness values of the matrix $I\left(t_{n}\right)$ and the next image $I\left(t_{n+1}\right)$ of the same location were selected as input data as an $18 \times 1$ column vector:

$$
\begin{aligned}
& I=\left[I\left(i-1, j-1, t_{n}\right) \quad I\left(i-1, j, t_{n}\right) \quad I\left(i-1, j+1, t_{n}\right) \quad I\left(i-1, j-1, t_{n+1}\right) \quad I\left(i-1, j, t_{n+1}\right) \quad I\left(i-1, j+1, t_{n+1}\right)\right. \\
& I\left(i, j-1, t_{n}\right) \quad I\left(i, j, t_{n}\right) \quad I\left(i, j+1, t_{n}\right) \quad I\left(i, j-1, t_{n+1}\right) \quad I\left(i, j, t_{n+1}\right) \quad I\left(i, j+1, t_{n+1}\right) \\
& \left.I\left(i+1, j-1, t_{n}\right) \quad I\left(i+1, j, t_{n}\right) \quad I\left(i+1, j+1, t_{n}\right) \quad I\left(i+1, j-1, t_{n+1}\right) \quad I\left(i+1, j, t_{n+1}\right) \quad I\left(i+1, j+1, t_{n+1}\right)\right]^{T}
\end{aligned}
$$

The input data were chosen in the format of $3 \times 3$ matrices so that the algorithm can act like a convolution mask filter for all pixels in the images. The brightness values between 0 and 255 were linearly transformed into the interval between 0 and 1 . The column vector was fed to the input layer. The hidden layer consisted of 14 nodes. The unit functions for input layer and hidden layer were logarithmic sigmoid transfer function.

$$
f\left(\text { net }_{j}\right)=\frac{1}{1+e^{- \text {net }_{j}}}
$$

There were 9 output nodes. The first output node represented no motion of the center pixel between the two successive images. The rest 8 nodes represented 8 directions of motion of the center pixel. The assumed output node was activated according to the motion direction of the center pixels between the two successive images (Table 1). The back propagation was Levenberg-Marquardt back propagation that updates weight and bias values according to Levenberg-Marquardt optimization. The back propagation is used to calculate the Jacobian of the performance with respect to the weight and bias variables. The learning function was gradient descent with momentum weight/bias learning function: 
$\mathrm{d} \mathbf{W}=m c * \mathrm{~d} \mathbf{W}$ prev $+(1-M C) * L R * g \mathbf{W}$

where weight change $d \mathbf{W}$ for a given neuron was calculated from the neuron's input, error, the weight or bias learning rate $L R$, and momentum constant $M C$, according to the gradient descent with momentum. The performance function was mean squared error performance function.

\subsection{Training data sets extracted from phantoms}

Phantom images were designed as two circular discs with diameters of 84 and 81 pixels. The discs were filled with representative pixels taken from a 10x10 rectangle on the left ventricular center $\left(i_{0}, j_{0}\right)$ to simulate the inhomogeneous contrast medium. For the systolic sequence, the larger circular disc was placed at time $t_{n}$ and the smaller disc was placed at time $t_{n+1}$ (Fig. 2). There is contraction of 1.5 pixels along the margin of the circular discs in the systolic sequence, simulating roughly the contraction of the left ventricle. The motion of contraction was assumed only around the margin of the discs of the left ventricle. The directions of motion were given by

$$
\phi(i, j)=\tan ^{-1}\left(\frac{i-i_{0}}{j-j_{0}}\right)
$$

The expected output node for each pixel could be derived by the direction of motion. For the diastolic sequence, the smaller disc was placed at time $t_{n}$ and the larger disc was placed at time $t_{n+1}$. There was expansion of 1.5 pixels along the margin of the circles in the sequence, simulating roughly the dilatation or relaxation of the left ventricle. There were total 3137

Table 1. The examples of training data sets taken from phantom images, in relation with their assumed directions of motion, motion vectors, and assumed output nodes.

\begin{tabular}{|c|c|c|c|}
\hline $\begin{array}{l}\text { Examples of training data sets } \\
\text { (for } 18 \text { input nodes) }\end{array}$ & $\begin{array}{l}\text { Directions of } \\
\text { motion }\end{array}$ & Motion vectors & $\begin{array}{l}\text { Assumed output } \\
\text { nodes }\end{array}$ \\
\hline$t_{n+1}$ & - & $(0,0)$ & 1 \\
\hline$t_{n+1}$ & $\rightarrow$ & $(1,0)$ & 2 \\
\hline$t_{n+1}$ & $\mathbf{y}$ & $(1 / \sqrt{2}, 1 / \sqrt{2})$ & 3 \\
\hline$t_{n+1}$ & $\downarrow$ & $(0,1)$ & 4 \\
\hline$t_{n+1}$ & $\boldsymbol{K}$ & $(-1 / \sqrt{2}, 1 / \sqrt{2})$ & 5 \\
\hline$\exists{ }^{t_{n+1}}$ & $\leftarrow$ & $(-1,0)$ & 6 \\
\hline$\theta t_{n+1}$ & $\mathbf{K}$ & $(-1 / \sqrt{2},-1 / \sqrt{2})$ & 7 \\
\hline$\exists t_{n+1}$ & $\uparrow$ & $(0,-1)$ & 8 \\
\hline$t_{n}=\rightleftarrows t_{n+1}$ & $\pi$ & $(1 / \sqrt{2},-1 / \sqrt{2})$ & 9 \\
\hline
\end{tabular}




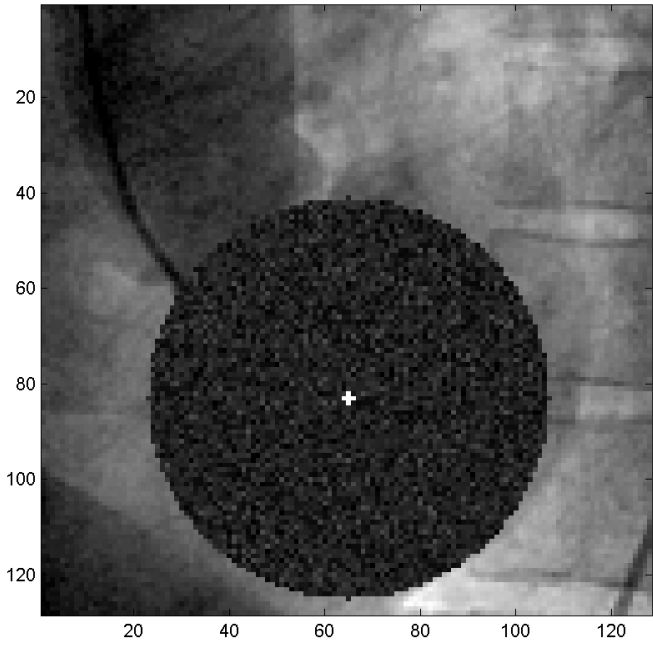

(a)

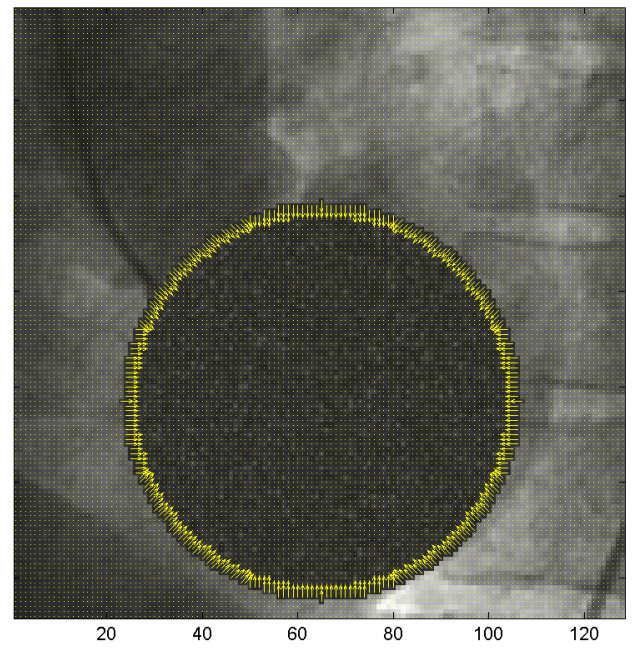

(b)

Figure 2. A phantom circular disc randomly filled with the representative pixels to simulate the left ventricle (a). Motion vectors were assumed along the margin of the contracting circular disc (b).

training data sets selected from the phantom images. The neural network was trained until a convergence of less than 0.01 was achieved. After training, the neural network can perform motion detection for the phantom images and the whole sequences of patient' angiocardiography. The computation was implemented by a personal computer with Pentium-IV $1.5 \mathrm{GHz}$ CPU and 768MB RAM, using MATLAB v6.1 (Mathworks, Inc. Massachusetts, U.S.A.) with neural network toolbox v4.0. The averaged training time cost about 7 hours.

\subsection{Motion detection on patients' angiocardiography}

The angiocardiography was done routinely with Philips Integris BH5000 (Philips Inc., U.S.A.). The LAO view images of the left ventricle were acquired by fluoroscopy. The cine movies were output onto DICOM III CD-ROM. The serial images were $512 \times 512$ matrices with frame rate of 30 frames per second. Three patients were selected. One patient had normal cardiac wall motion over the left ventricle. The other two patients had heart disease with abnormal cardiac wall motion. For general patients, there are usually 20 to 30 frames of cine angiocardiographic images in each heart cycle. The preprocessing of the cine images were gray scale modification and re-sampling to reduce the matrices to $128 \times 128$. The gray scale modification was linear and made the gray scale of left ventricular contrast medium to be about $0.08(20 / 255)$ and the brightest area to be 1 . After pre-processing, patients' images were fed to the trained neural network for motion detection. The outputs of the motion detection were displayed in motion vector fields superimposed on the original image at time $t_{n}$. The neural network can perform motion detection for all series of the sequential images at any time $t_{n}$ and $t_{n+1}$. The motion detection for the whole sequence of systolic and diastolic motion cost about 30 minutes. 


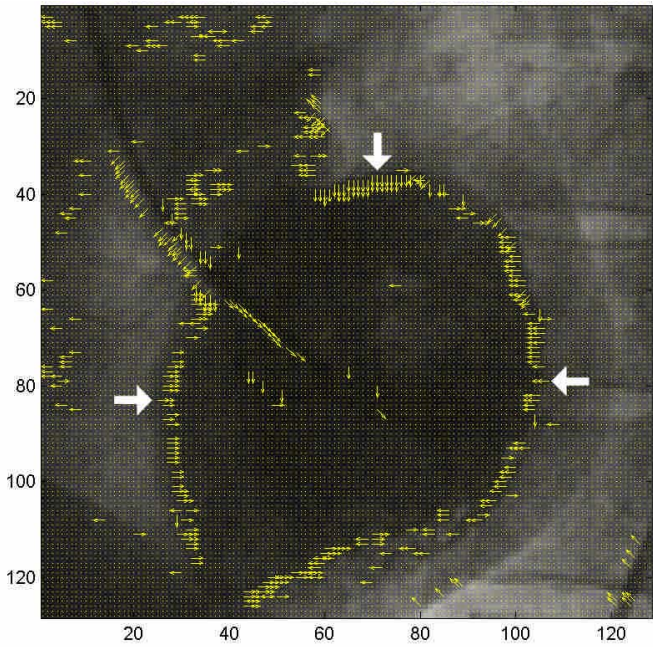

(a)

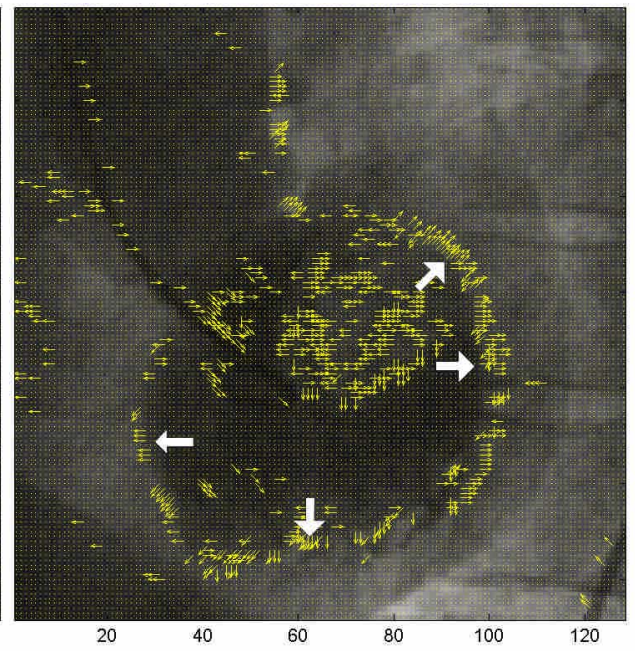

(b)

Figure 3. Motion vectors of normal cardiac wall motion. Centripetal motion (arrows) was observed in the systolic sequence (a), and centrifugal motion (arrows) in the diastolic sequence (b).
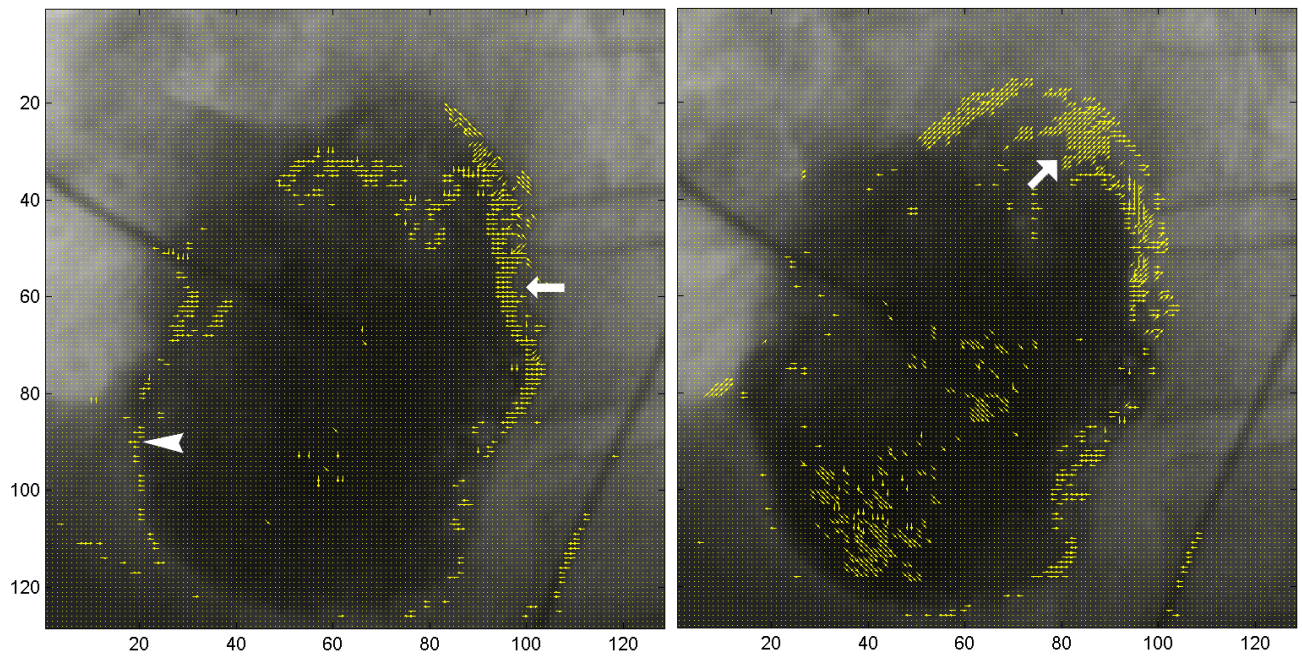

Figure 4. Motion vectors of a patient with abnormal cardiac wall motion. Inconsistent centrifugal motion (arrow head) was observed over the septal wall in the systolic sequence.

\section{Results}

The outputs for motion detection on the phantom images showed good performance with $100 \%$ accuracies for both systolic and diastolic phantom sequences. The result was displayed in the format of vector fields superimposed on the phantom image and showed the same as Figure 2b.

The results of motion detection on patients' angiocardiography were displayed in the formats of vector fields. For the patient with normal cardiac wall motion, the systolic 
sequence showed normal centripetal motion over the lateral wall, anterior wall, and septum (Fig. 3a). The motion of the catheter was also detected and shown. In the diastolic sequence there were normal centrifugal motion over the anterolateral wall, lateral wall, inferior wall, and septum (Fig. 3b). There was turbulent flow with the contrast medium observed over the upper part of the left ventricle.

Motion detection the diseased patients with abnormal cardiac wall motion showed no motion (akinesia) over the anterior wall and inferior wall, and some inconsistent centrifugal motion (dyskinesia) over the septum (arrow head in Fig. 4). In the diastolic sequence there was no motion observed over anterior wall, septum, and inferior wall (Fig. 4). The results of motion detection by the neural network can reflect akinesia and dyskinesia of the abnormal cardiac wall motion. The above results were consistent with the ultrasonographic and angiocardiographic findings, which were made by the experienced cardiologists.

\section{Conclusion and discussions}

The construction of phantom images and selection of training data sets are important for the performance of the back propagation neural network. The more the phantom images resemble the original images, the better the performance. Because the left ventricle of a real patient is never ideally circular, extracting training data directly from the boundaries of the left ventricle cannot easily be implemented. Constructing the phantom images by two circular discs was a more intuitive and simpler way. The convergence of the training process and good performance revealed the appropriateness of the phantom construction. The fuzzy reasoning and the fault tolerance of the neural network allowed the detection of more complex motion of real patients' images than the ideal phantom images.

The performance of motion detection by the back propagation neural network was perfect for the phantom images. The performance of motion detection by the neural network was acceptably good for patients' images. The motion detection over the areas of normal wall motion and akinesia showed acceptably good results. The results were obvious by visual inspection on the vector fields. The study showed that back propagation neural network was useful in the analysis of cardiac wall motion on angiocardiography. The study suggests that other cardiac imaging modalities, such as cardiac cine MR or electron beam CT may gain useful information by this method.

\section{References}

[1] A.D. Bimbo, P. Nesi, and J.L.C. Sanz, "Optical flow computation using extended constraints", IEEE Transactions on Image Processing, 5, 1996, pp. 720-739.

[2] Tekalp, A.M., Digital video processing, Prentice Hall, Upper Saddle River, NJ, 1995.

[3] E. Atsumi, M. Takagi, and K. Yokosawa, "Local motion detection by hierarchical neural network", Systems and Computers in Japan, vol. 25, No. 11, 1994, pp. 24-35.

[4] T. Roska, T. Boros, A. Radvanyi, P. Thiran, and L.O. Chua, "Detecting moving and standing objects using cellular neural networks", International Journal of circuit theory and applications, vol. 20, 1992, pp. 613-628.

[5] Y.C. Shiau, H.H. Chou, S.H. Chu, and T.S. Kuo, "Cardiac wall motion detection by neural network analysis on Tc-99m MIBI myocardial perfusion gated single photon emission computed tomography", Proc. IEEE EMBS Asian-Pacific Conf. on Biomedical Engineering 2003, Kyoto, Japan, 2003, p. 13.

[6] R.D. White and R.M. Setser, "Integrated approach to evaluating coronary artery disease and ischemic heart disease", American Journal of Cardiology, 90 (10C), 2002, pp. 49L-55L. 